Journal Club

Editor's Note: These short, critical reviews of recent papers in the Journal, written exclusively by graduate students or postdoctoral fellows, are intended to summarize the important findings of the paper and provide additional insight and commentary. For more information on the format and purpose of the Journal Club, please see http://www.jneurosci.org/misc/ifa_features.shtml.

\title{
Neuroinflammation Mediates Synergy between Cerebral Ischemia and Amyloid- $\beta$ to Cause Synaptic Depression
}

\author{
(1)Walter Swardfager, ${ }^{1,2}$ () Madelaine Lynch, ${ }^{1,3}$ Sonam Dubey, ${ }^{1,3}$ and $\odot$ Paul M. Nagy ${ }^{1}$ \\ ${ }^{1}$ Sunnybrook Research Institute, Toronto, Ontario, M4N 3M5 Canada, and ${ }^{2}$ Department of Pharmacology and Toxicology, ${ }^{3}$ Department of Laboratory \\ Medicine and Pathobiology, University of Toronto, Toronto, Ontario, M5S 2J7 Canada \\ Review of Origlia et al.
}

Alzheimer's disease $(\mathrm{AD})$ is the most common cause of dementia, and autopsy series suggest that cerebrovascular disease is a highly prevalent contributor. Risk factors for the development of $\mathrm{AD}$ tend to be related to vascular disease, including dyslipidemia, diabetes, hypertension, and arteriosclerosis of blood vessels large and small, all of which impair circulation and microcirculation, and may lead to cerebral ischemia. In support of a link between ischemia and amyloid pathology, a recent clinical neuroimaging study found that disease of the small cerebral vessels preceded the deposition of amyloid- $\beta$ (Grimmer et al., 2012). For this reason, the mechanisms by which ischemia exacerbates the progression of $\mathrm{AD}$ are of interest, and animal models that include a combination of ischemia and elevated amyloid- $\beta$ may be useful in understanding the molecular events that contribute to the progression of human $\mathrm{AD}$.

In their paper, Origlia and colleagues (2014) used electrophysiological recordings from mouse entorhinal cortical slices to demonstrate that oxygen and glucose deprivation (OGD) depressed synaptic transmission, and that this effect was po-

Received Aug. 1, 2014; revised Aug. 27, 2014; accepted Aug. 30, 2014.

W.S. acknowledges postdoctoral support from the Canadian Institutes of Health Research.

Correspondence should be addressed to Dr. Walter Swardfager, Sunnybrook Research Institute, 2075 Bayview Avenue, Toronto, Ontario, M4N 3M5 Canada. E-mail: wlswardf@gmail.com.

DOI:10.1523/JNEUROSCI.3196-14.2014

Copyright $\odot 2014$ the authors $\quad 0270-6474 / 14 / 3413571-03 \$ 15.00 / 0$ tentiated by the addition of amyloid- $\beta$. The synergy between amyloid and OGD was confirmed using a transgenic mouse model in which expression of humanized amyloid precursor protein (hmAPP) caused overproduction of amyloid- $\beta$. In these animals, OGD increased amyloid- $\beta$ production and an amyloid- $\beta$-lowering $\gamma$-secretase inhibitor ameliorated synaptic depression in cortical slices following OGD. Thus, consistent with previous studies, it was demonstrated that amyloid- $\beta$ could exacerbate postischemic neuronal dysfunction.

In their previous work, Origlia and colleagues found that amyloid- $\beta$ activation of the receptor for advanced glycation endproducts (RAGE) induced IL- $1 \beta$ release in cortical slices (Origlia et al., 2010). Therefore in the present study, they examined RAGE and IL- $1 \beta$ as potential mediators of the deleterious synergy between ischemia and amyloid- $\beta$. They showed that a RAGE inhibitor, or microglial-specific genetic inactivation of RAGE, protected against deficits in synaptic function elicited by OGD in the presence of amyloid- $\beta$. They demonstrated that OGD elevated IL- $1 \beta$ and that blocking the IL-1 receptor rescued synaptic function otherwise impaired by OGD, implicating IL- $1 \beta$ as a mediator of the OGD effect on neuronal dysfunction. Collectively, the findings suggest that neuroinflammation may be at the crux of ischemia-induced deficits in synaptic transmission, to which amyloid- $\beta$ over- production may make AD brains particularly susceptible.

The authors propose that ischemia leads to extracellular amyloid- $\beta$, which leads to neuroinflammation and synaptic dysfunction in a "self-maintained loop" (Origlia et al., 2014, their Fig. 7). They demonstrate that microglial RAGE responds to amyloid- $\beta$ and contributes to IL- $1 \beta$ production, leading to synaptic depression, but how these signals become amplified remains unclear. One possibility is that neuroinflammation might lead to increased amyloid- $\beta$ production, increasing RAGE activation. Amyloid- $\beta$ overproduction is primarily neuronal in this mouse model (Liu et al., 2004) and previous studies have suggested that inflammatory signals mediated by NF- $\kappa \mathrm{B}$ can promote amyloid- $\beta$ synthesis in neurons that have been previously exposed to amyloid- $\beta$ (Bourne et al., 2007). Therefore, it is possible that neuroinflammation downstream of RAGE could contribute to neuronal amyloid- $\beta$ synthesis. However, while the present experiments strongly implicate IL- $1 \beta$ as a distal mediator of neuronal dysfunction, they do not establish a role of IL- $1 \beta$ as a mediator of a feedforward loop (Fig. 1). To do this, it would be necessary to assess whether IL-1 receptor blockade also decreases amyloid- $\beta$ production during OGD.

Alternatively, RAGE activation might increase amyloid- $\beta$ production by a mechanism not directly related to IL- $1 \beta$. Although Origlia and colleagues (2014) 


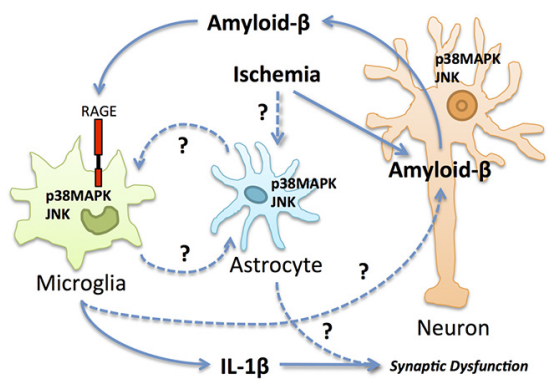

Figure 1. Possible relationships between ischemia, amyloid- $\beta$, and neuroinflammation. Origlia and colleagues (2014) demonstrated that ischemia can induce amyloid- $\beta$ synthesis, microglial RAGE-dependent JNK activation, p38MAPK activation, and IL-1 $\beta$ release that results in synaptic dysfunction. They suggest a feedforward loop whereby the presence of amyloid- $\beta$ during ischemia exacerbates neuroinflammation, which promotes additional amyloid- $\beta$ production. To close this loop, further work should investigate the contributions of microglial, astrocyte, neuronal, and endothelial RAGE, and neuroinflammation to amyloid- $\beta$ overproduction during OGD. While the molecular events leading to IL-1 $\beta$ production required JNK and p38MAPK activation, the specific cell types expressing these activated kinases remain to be determined. Astrocytes and neurons, as well as microglia, activate JNK and p38MAPK after ischemia (Wu et al., 2000) and any of these cell types could be involved in a feedforward loop that supports IL- $1 \beta$ release from microglia and postischemic synaptic dysfunction. Interactions established in the study (solid lines) and interactions suggested in previous studies (dashed lines) that could support a feedforward mechanism are shown.

report that genetic inactivation of microglial RAGE did not suppress amyloid- $\beta$ production in hmAPP mice under ischemic conditions, they do not present for comparison the levels from hmAPP mice with intact microglial RAGE (Origlia et al., 2014, their Fig. 3C). A comparison of amyloid- $\beta$ concentrations after OGD between mice lacking microglial RAGE and those with intact microglial RAGE would be needed to determine whether microglial RAGE increases amyloid- $\beta$ production during ischemia. Previously, a RAGE inhibitor was demonstrated to lower amyloid- $\beta$ in amyloid overproducing mice (Deane et al., 2012), but the inhibitor was not specific to microglial RAGE and it was not applied during OGD. In those experiments, RAGE expressed on other cell types may have increased amyloid- $\beta$ synthesis. In short, while the results of Origlia and colleagues (2014) show that neuronal amyloid- $\beta$ and microglial RAGE potentiate synaptic depression after OGD, they do not establish whether amyloid- $\beta$, microglial RAGE, or IL- $1 \beta$ is sufficient to initiate or sustain a self-maintained loop.

Next, using small-molecule inhibitors, Origlia and colleagues (2014) probed the possible roles of the intracellular stress- related kinases JNK and p38MAPK, based on their previous observations that these kinases are involved in RAGE signaling pathways (Origlia et al., 2010). They demonstrated that activation of JNK and p38MAPK are necessary to support IL- $1 \beta$ production and synaptic depression in response to OGD (Origlia et al., 2014, their Fig. 7); however, these experiments do not identify the particular cell types in which JNK and p38MAPK activations contribute to IL-1 $\beta$ production. Activation of JNK and p38MAPK occurs in neurons, microglia, and other cell types in response to ischemia; notably, astrocytes activate JNK and p38MAPK within 10 min of ischemia (Wu et al., 2000) and they might also participate in a feedforward loop that ultimately determines the extent of microglial IL-1 $\beta$ production and neuronal dysfunction (Fig. 1). In fact, astrocytes activated by ischemia engage in crosstalk with microglia; cytokines produced by ischemic astrocytes modulate microglial cytokine release, which feeds back to modify the release of soluble factors from astrocytes (Zamanian et al., 2012). Furthermore, astrocyte-derived soluble factors have been implicated in neuronal tau hyperphosphorylation and neuronal loss due to amyloid- $\beta$, and they might directly modulate synaptic depression after OGD.

In their final set of experiments, comparing wild-type slices to hmAPP slices with and without genetic deletion of microglial RAGE under OGD, a curious puzzle emerged with regard to activation of the stress-related kinases. In wild-type animals, p38MAPK but not JNK activation was dependent on microglial RAGE during ischemia, whereas in amyloid- $\beta$ overexpressing mice, both $\mathrm{p} 38 \mathrm{MAPK}$ and JNK activation depended on microglial RAGE. In hmAPP mice, gene expression may have been altered by amyloid- $\beta$, setting the stage for RAGE dependence; amyloid- $\beta$ can accumulate intracellularly and affect responses to extracellular stimuli (Bourne et al., 2007). In this last set of experiments, it does not appear that exogenous amyloid- $\beta$ was added to the wild-type condition during OGD. It would be interesting to determine whether JNK activation is dependent on microglial RAGE in the wild-type mice in the presence of amyloid- $\beta$, or whether some other feature of the hmAPP model is driving this difference, such as changes in gene expression due to long-standing amyloid- $\beta$ elevations. For instance, it has been suggested that activation of RAGE by amyloid- $\beta$ can increase microglial RAGE expression, which may support a putative feedforward loop, as discussed above.
However, in the present study, RAGE expression levels were not compared between wild-type and hmAPP cortical slices. Further work might seek to discern the patterns of RAGE, activated JNK and activated p38MAPK specifically in microglia, astrocytes and neurons by immunocytohistochemistry, or flow cytometry in tissue from wild-type and hmAPP mice after ischemia.

Future studies might explore relationships between RAGE activation and tau hyperphosphorylation, a second hallmark $\mathrm{AD}$ pathology that increases in ischemia models and is closely related to both neuronal dysfunction and neuroinflammation (Wen et al., 2004). Notably, IL-1 $\beta$ release from microglia induces hyperphosphorylation and aggregation of somatodendritic tau in neurons via the p38MAPK pathway (Bhaskar et al., 2010). Therefore, it would be useful to assess whether this too might be prevented by RAGE inhibition or IL-1 receptor blockade.

The findings of Origlia and colleagues (2014) are intriguing and timely. Although amyloid- $\beta$ is an established hallmark of $\mathrm{AD}$ pathology, to date, antiamyloid therapies have failed in clinical trials. Similarly, ample evidence supports a role of inflammation in the pathophysiology of human $\mathrm{AD}$ but disease-modifying anti-inflammatory therapies remain elusive. Moreover, a RAGE inhibitor trial was recently discontinued when subjects randomized to receive a high dose of the inhibitor experienced adverse effects and poorer cognitive outcomes, although a lower dose showed potential benefit of borderline significance (Galasko et al., 2014). The failure of trials based on these targets suggests the need to further dissect the proposed loop of reciprocal signal amplification to better understand this synergy and identify new targets (Fig. 1). The identification of IL- $1 \beta$ as a mediator of RAGE-dependent synaptic dysfunction during ischemia suggests that soluble IL- $1 \beta$ receptors, which can cross the blood brain barrier, might be tried as $\mathrm{AD}$ therapeutics but other inflammatory mediators may also be important. Animal models that combine amyloid pathology and ischemia may continue to provide helpful insights.

\section{References}

Bhaskar K, Konerth M, Kokiko-Cochran ON, Cardona A, Ransohoff RM, Lamb BT (2010) Regulation of tau pathology by the microglial fractalkine receptor. Neuron 68:19-31. CrossRef Medline

Bourne KZ, Ferrari DC, Lange-Dohna C, Rossner S, Wood TG, Perez-Polo JR (2007) Differential regulation of BACE1 promoter activity by nu- 
clear factor-kappaB in neurons and glia upon exposure to beta-amyloid peptides. J Neurosci Res 85:1194-1204. CrossRef Medline

Deane R, Singh I, Sagare AP, Bell RD, Ross NT, LaRue B, Love R, Perry S, Paquette N, Deane RJ, Thiyagarajan M, Zarcone T, Fritz G, Friedman AE, Miller BL, Zlokovic BV (2012) A multimodal RAGE-specific inhibitor reduces amyloid beta-mediated brain disorder in a mouse model of Alzheimer disease. J Clin Invest 122:1377-1392. CrossRef Medline

Galasko D, Bell J, Mancuso JY, Kupiec JW, Sabbagh MN, van Dyck C, Thomas RG, Aisen PS, Alzheimer's Disease Cooperative Study (2014) Clinical trial of an inhibitor of RAGEAbeta interactions in Alzheimer disease. Neurology 82:1536-1542. CrossRef Medline

Grimmer T, Faust M, Auer F, Alexopoulos P,
Förstl H, Henriksen G, Perneczky R, Sorg C, Yousefi BH, Drzezga A, Kurz A (2012) White matter hyperintensities predict amyloid increase in Alzheimer's disease. Neurobiol Aging 33:2766-2773. CrossRef Medline

Liu BH, Wang X, Ma YX, Wang S (2004) CMV enhancer/human PDGF-beta promoter for neuron-specific transgene expression. Gene Therapy 11:52-60. CrossRef Medline

Origlia N, Bonadonna C, Rosellini A, Leznik E, Arancio O, Yan SS, Domenici L (2010) Microglial receptor for advanced glycation end product-dependent signal pathway drives beta-amyloid-induced synaptic depression and long-term depression impairment in entorhinal cortex. J Neurosci 30:11414-11425. CrossRef Medline

Origlia N, Criscuolo C, Arancio O, Yan SS, Do- menici L (2014) RAGE inhibition in microglia prevents ischemia-dependent synaptic dysfunction in an amyloid-enriched environment. J Neurosci 34:8749-8760. CrossRef Medline

Wen Y, Yang S, Liu R, Simpkins JW (2004) Transient cerebral ischemia induces sitespecific hyperphosphorylation of tau protein. Brain Res 1022:30-38. CrossRef Medline

Wu DC, Ye W, Che XM, Yang GY (2000) Activation of mitogen-activated protein kinases after permanent cerebral artery occlusion in mouse brain. J Cereb Blood Flow Metab 20: 1320-1330. CrossRef Medline

Zamanian JL, Xu L, Foo LC, Nouri N, Zhou L, Giffard RG, Barres BA (2012) Genomic analysis of reactive astrogliosis. J Neurosci 32: 6391-6410. CrossRef Medline 